\section{SENSITIVITY OF THE EAR}

$\mathrm{T}$ HE difficulty in securing accurate values of the minimum stimulus that the ear can detect is great. In the first place there are differences in the hearing mechanisms of various individuals tested. Except for those with some definite hearing defect, these differences largely depend on age; as one grows older the response of the ear becomes less, chiefly at the higher frequencies. Measurements of minimum hearing stimulus thus vary with the age of those being tested. Another difficulty is in measuring the minimum stimulus. According to an article by W. A. Munson (Beill Lab. Rec., 21, No. 10; June 1943) two references have been widely used. One is the rms. pressure of the sound wave at a point in a free sound field where the listener's ear will later be placed. This is the simpler of the two, and is particularly applicable to studies of the usual mode of hearing, that is, without the use of telephone receivers or similar devices. The other is the pressure at the ear-drum. This reference avoids uncertainty as to how the pressure, measured in the air previously to the listening test, would be modified at the ear-drum by the head and ear of the listener, and is particularly useful in studying the mechanism of hearing. It involves great technical difficulties, however, since the space in front of the ear-drum is small and almost inaccessible.

Regardless of which of these two methods is used, the pressure involved is very minute-less than 1/1,000 dyne/sq. cm. A pressure of this order of magnitude distributed over the ear-drum results in a force of the order of only $1 / 10^{8} \mathrm{oz}$. As an organ for detecting pressure changes, the ear is thus far more sensitive than our tactile organs, since a touch of this lightness would be imperceptible. Even at the upper limit of hearing, the force is only some thousandths of an ounce. Vibrating pressures greater than this become tactable rather than audible and result in the sensation of pain. Any air wave with an rms. pressure above the threshold value enters our consciousness as sound, and the sound becomes louder the higher the pressure. Since it is the loudness rather than the pressure that we are directly conscious of, some scale for measuring loudness is also desirable.

The results of a large amount of work were summarized some years ago by Fletcher and Munson and it was decided to use the loudness of a 1,000-cycle tone as a reference. This frequency is widely used as a test tone throughout the Bell System, and it has the advantage of being very close to the frequency range for which the ear is most sensitive. Since in general the relationship between the magnitude of a sensory response and its stimulus is logarithmic, it seemed desirable to use a logarithmic scale, and for convenience the loudness-level of a 1,000-cycle tone was arbitrarily taken to be the intensity level. Loudness-level at any other frequency or combination of frequencies is taken to be equal to the intensitylevel of a 1,000-cycle tone that sounds equally loud. Although loudness-level is measured on a logarithmic scale, and thus corresponds in magnitude to a decibel scale, the unit is called a phon instead of a decibel to indicate that the thing measured is basically different from power.

At 1,000 cycles the threshold of hearing in a free field is just about at 0 intensity-level, which also justifies making the loudness-level and intensity-level equal by definition at this frequency. At 1,000 cycles, therefore, an increase in intensity-level results in an equal increase in loudness-level. This equality is found not to exist at other frequencies, however.

To the question: How little do we hear? the only safe answer is : it all depends. What we hear is a sequence of periodic pressure changes in the air, but how small the pressure changes may be, given a normal young ear, depends on the rapidity of the changes. The ear is most sensitive when the pressure cycles are occurring at rates between 1,000 and 5,000 times per second, since we can then detect changes of less than a thousandth of a dyne/sq. cm. At both higher and lower frequencies, the sensitivity falls off. The studies that have determined these minimum perceptible pressure changes, and the many others that have established units of measurement and adequate techniques and apparatus, have provided the foundations for the extensive studies of hearing that have been carried on in the Laboratories.

\section{CERAMICS FOR HIGH-FREQUENCY INSULATION}

A CCORDING to an article by M. D. Rigterink (Bell Lab. Rec., 21, No. 9; May 1943) electrical poreelains which are satisfactory and useful materials for low-frequency currents have been replaced for high-frequency currents by new ceramic materials which have far smaller power factors. This replacement becomes absolutely essential at elevated temperatures such as are encountered in power vacuum tubes in which the insulator operates almost red hot. The power factor of most ceramics increases very markedly with increase in temperature, this causing more conversion of electrical energy into heat and producing a cumulative effect which may lead to a breakdown in any but the very best materials.

The most widely used of the improved ceramics are the steatites, this term now being applied to all synthetic ceramic materials which are prepared with talc as the principal raw material and which have magnesium metasilicate as the principal crystalline phase. Steatite bodies are prepared from mixtures of 60 per cent or more talc, 30 per cent or less kaolin, and the remainder usually of alkali or alkaline earth oxides or compounds that will decompose to give these fluxing oxides. The formed ware is heated from 1,200 to $1,400^{\circ} \mathrm{C}$. on a controlled, heating and cooling cycle. During this heat treatment the fluxing oxides combine with part of the magnesia, alumina, and silica given by the talc and the clays to form glasses which in turn disselve more of these constituents as the temperature rises. In addition to glass formation, chemical reactions take place between the solids and between the solids and liquid glasses; crystallizations also occur as the temperature is lowered, and finally crystalline transformations are produced. These complex changes cause the mixture to shrink and to become the hard, dense material known as a steatite ceramic.

Dielectric properties of the steatite ceramics vary considerably, depending on the amount, kind and proportions of fluxing oxides that are used in their preparation. The alkalis are particularly detrimental, and the alkaline earth oxides appear to be especially beneficial. Other variations are caused by the grinding and mixing of the raw materials, the firing cycles, and the nature and the amount of impurities present. All the steatites have unusually 